\title{
Definition of composite profiles for the needs of a dynamic analysis of a rail vehicle
}

\author{
Vladimír Hauser ${ }^{1, *}$, Juraj Gerlici ${ }^{1}$, Kateryna Kravchenko ${ }^{1}$, Tomáš Lack ${ }^{1}$, Mária Loulová1, \\ and Olha A. Potapenko ${ }^{2}$ \\ ${ }^{1}$ Department of Transport and Handling Machines, Faculty of Mechanical Engineering, University of \\ Zilina, Univerzitna 8215/1, 01026 Zilina, Slovak Republic \\ ${ }^{2}$ Department of Railway and Road Transport and Handling Machines, Educational and Scientific \\ institute of Transport and Logistics, V. Dahl East Ukrainian National University, Centralnyj av. 59a, \\ 93406 Ceverodoneck, Ukraine
}

\begin{abstract}
When modelling the dynamics of a rail vehicle ride, one of the important input parameters is the profile geometry of the wheel and rail treads. Commonly available simulation programs have databases containing some standardized profiles, or allow the profile to be added to the database. However, complications arise when a specific profile is required in the simulation analysis. Its geometry must be entered in the form of coordinates of the knot points that can be used to render the curve and thus define the desired profile. Simulation programs, however, do not have the ability to efficiently generate these coordinates. Since this is a lengthy process, the author team has created a program to define this profiles, based on their common drawing documentation. This program allows to create even large amount of profiles needed for variable rail treads definitions of crossings and switch points by a smart way.
\end{abstract}

Keywords: composite rail profiles, specific track section, tramcar ride analysis

\section{Introduction}

Simulation analysis of vehicles ride dynamics currently plays a key role in the development of modern transport. Computational programs make it possible to execute a multi-body multiphysical analysis of the solved task efficiently. They help minimize the financial and time-consuming requirements of the vehicle development. They also allow to predict the behavior of the designed mechanical structures or a whole vehicle in different situations, without the need to build a prototype. They help the engineer to decide for the most appropriate option from amongst possible solutions. Performing the tests prescribed by standards in real conditions makes it possible to achieve a high match of the measured values on the measuring devices with the values expected based on the simulation analysis [1-4].

\footnotetext{
* Corresponding author: vladimir.hauser@fstroj.uniza.sk

Reviewers: Alžbeta Sapietová, Michat Śledziński
} 
In practice, as well as in the modeling of the dynamics of rail vehicles, one of the important input parameters is the geometry of the wheel and rail tread profile [5-8]. Commonly available simulation programs for such analysis have databases of certain components, including some standardized wheel and rail profiles or allow these profiles to be added to the database. In this case, it is necessary to enter the geometry of the profile in the required form necessary to ensure compatibility with the structure of the inputs of the computational software [9-12], that is, in the form of coordinates of the points lying on its surface in the plane of a cross section. A curve interconnecting these points, is defined during the calculation, by which the profile in the simulation analysis is defined. The profile serving as input for the computational program is therefore stored in the form of text files containing, among other parameters, in particular the nodal point coordinate sequence in the desired form.

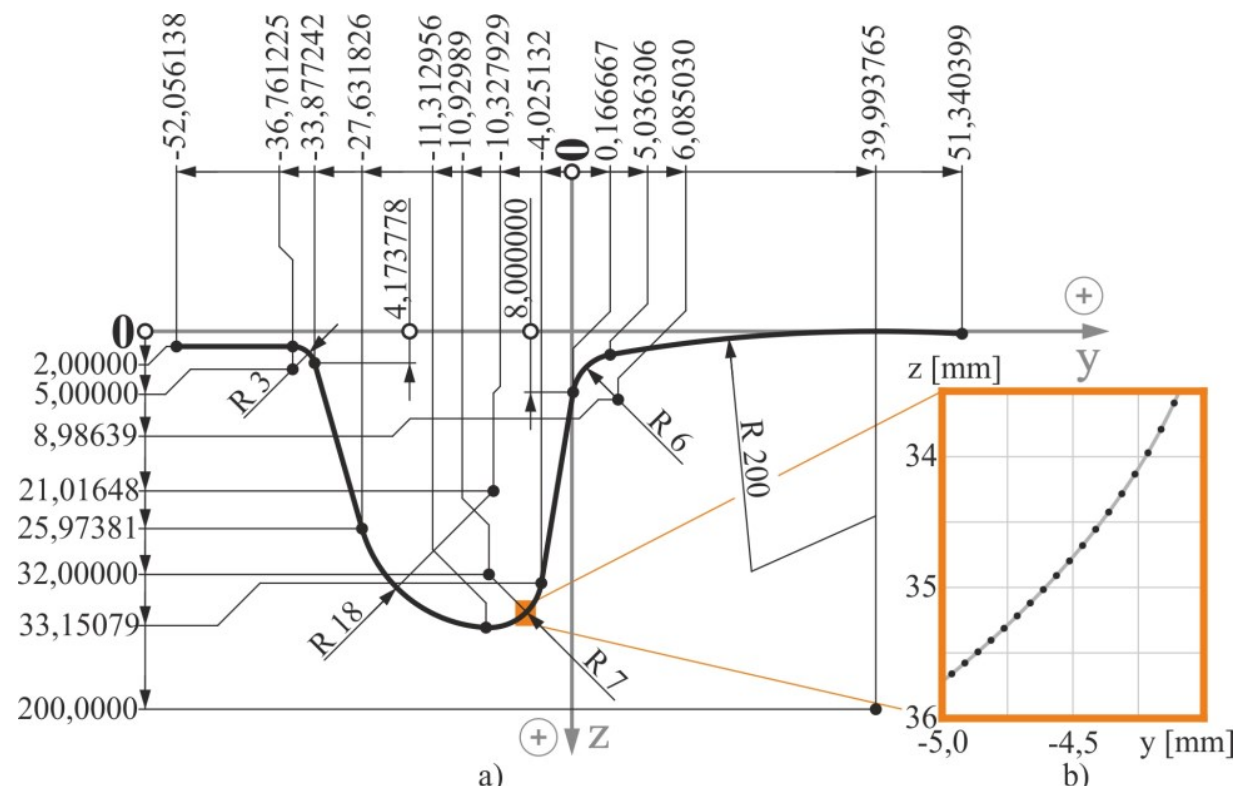

Fig. 1. a) Drawing documentation of the profile, b) Graphical representation of node point position for simulation purposes

For the simulation itself, it is possible to use a profile from the computation program database or a real profile, the geometry of which has been measured using modern measurement devices that, as output, give a sequence of points coordinates lying on the surface of a measured profile, which is highly compatible with the required input format of the calculation program.

However, complications occur when simulation analysis requires a specific profile to be defined only through its drawing documentation. Simulation programs do not have the ability to efficiently generate the necessary coordinates based on drawing documentation. In this case, it is necessary to re-calculate the coordinates of the nodal points forming the profile by using analytic expressions of the individual segments forming the geometry of the profile. For a correct expression of the required geometry, it is necessary to calculate coordinates of approximately 200 to 3000 points, depending on the complexity of the profile, to be evenly distributed over its functional surface.

The undisputed advantage of simulation programs is the ability to analyze a vehicle's response to ride on specific track sections, such as tongue rails and point frogs, rail junctions, or curved sections of the track characterized by significant wear, where a variable 
geometry of one or both rails is necessary to be defined in the simulation model. For its creation, tens to hundreds of profiles that are translated during the simulation with a Bezier surface defining the examined section of the respective section of the rail.

\section{Motivation}

In the past, the team of authors has been devoted to designing a specific arc of a small radius tramline track with the intention of minimizing the force effects of the passing vehicle on the track, thanks to the targeted use of variable geometry rails and a wheelset equipped with a secondary wheel tread profile [10] as shown in Fig. 2. One of the first steps necessary to perform the simulation analysis of the dynamics of the tramline vehicle run and to obtain an overview of the events taking place in the wheel and rail contact was the definition of the wheel and rail profiles.
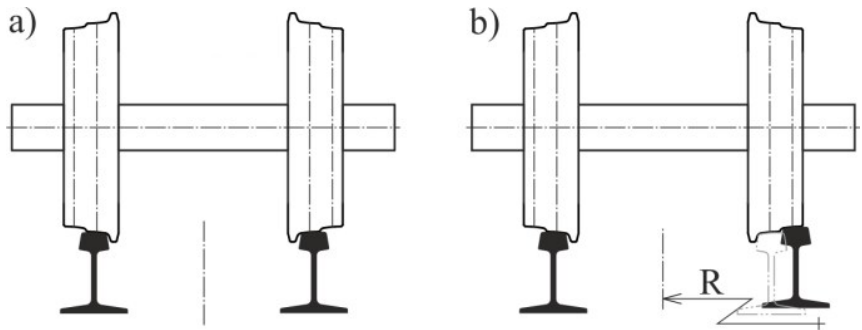

Fig. 2. The proposed solution for wheelset ride a) in usual track, b) in a small radius track curve

As our goal was to verify the idea described in Fig. 2 [10], we have experienced the need to speed up the creation of larger amount of desired profiles in the required shape and structure. For this purpose, a program has been created to automate this process to a certain extent [13] and to define profiles in the required shape based on their drawing documentation. The program and the methodology used for the generation of the coordinates has been awarded the Certificate of Originality and Copyright Registration of the Work "Coordinate Definition of Object Profile Geometry Points" No 73363 [9].

One of the effects taking place in the proposed process of ride through an arc of the track is transfer of the wheel-rail contact point outside the original wheel tread. Our goal was to analyze this phenomenon and to verify or compare it with a similar phenomenon occurring when passing through a frog of a city railway point, where the contact point is temporarily moved away from the wheel tread as shown in Fig. 3.
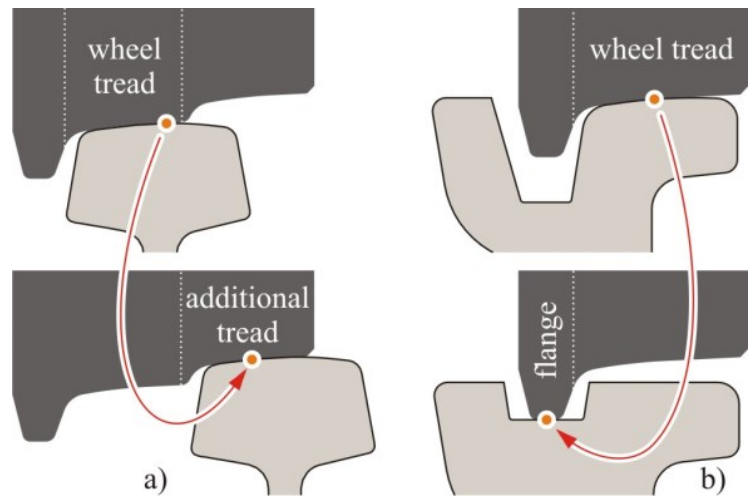

Fig. 3. Transfer of the contact point a) according to the proposed solution for the secondary wheel tread, b) in normal operation when passing through the frog with the wheel flange 
The point frog represents a specific section of the rail of variable geometry. In order to correctly describe it using a Bezier surface and subsequent processing in the simulation program, it was necessary to create more than 100 profiles of its cross-section in a $500 \mathrm{~mm}$ long section. The team of authors therefore proceeded to modify the proven profile generation program [9] to enable the creation of sections of a point rail on the basis of a pair of partially overlapping profiles.

\section{Methodology of generating coordinates}

A simplified scheme describing the profile creation algorithm in the desired form is shown in Fig. 5. The basic input for the program are the values read from the profile drawing (Fig. 1) and the required nodal point coordinates distance measured in the lateral direction. In case description of the point where profiles are crossing is required, it is also necessary to specify dimensions of the crossing profile, crossing angle and distance from the crossing point as shown in Fig. 4.

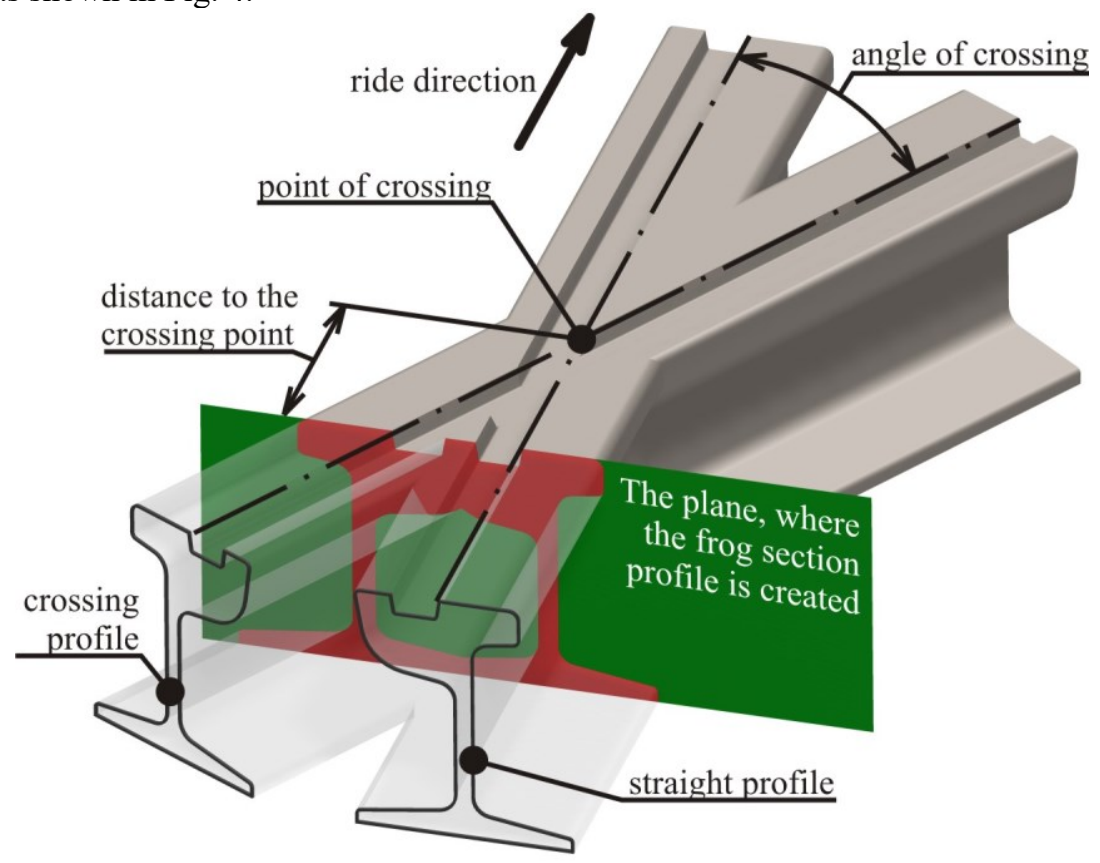

Fig. 4. Composite profile formed by crossing of the rail belts in the heart of the crossings of the city tracks

Once these values have been entered, the program analyzes the entered inputs and starts the y-coordinate generating cycle. It is continuously checked, which of the specified segment profiles this y-component belongs to. Based on its classification, the program creates analytical equations describing the geometry of the segment in the form (1) for an abscissa, (2) for a concave circular arc, (3) for a convex circular arc. In case of a crossing profile, the relationships (2) and (3) are changed to the form (4) and (5) as the crossing profile is projected into the plane of a perpendicular cross-section of the straight profile, thereby causing the circle segment to scarf to an elliptical segment. 


$$
\begin{gathered}
z=\frac{z_{z}-z_{k}}{y_{z}-y_{k}} \cdot y+\left(z_{z}-\frac{z_{z}-z_{k}}{y_{z}-y_{k}}\right) \cdot y_{z} \\
z=\sqrt{r^{2}-\left(y-S_{y}\right)^{2}}+S_{z} \\
z=-\sqrt{r^{2}-\left(y-S_{y}\right)^{2}}+S_{z} \\
z=\sqrt{r^{2}-\frac{r^{2}\left(y-S_{y}\right)^{2}}{\left(\frac{r}{\cos \alpha}\right)^{2}}}+S_{z} \\
z=-\sqrt{r^{2}-\frac{r^{2}\left(y-S_{y}\right)^{2}}{\left(\frac{r}{\cos \alpha}\right)^{2}}}+S_{z}
\end{gathered}
$$

where:

$z$ - the $\mathrm{z}$-coordinate of the nodal point,

$z_{z}-\mathrm{z}$-coordinate of the beginning of the abscissa-shaped segment,

$z_{k}$ - the exponential coordinate of the abscissa-shaped segment end,

$y_{z}-\mathrm{y}$-coordinate of the beginning of the abscissa-shaped segment,

$y_{k}-\mathrm{y}$-coordinate of the abscissa-shaped segment end,

$y-\mathrm{y}$ coordinate of the nodal point,

$r$ - radius of the circle segment,

$S_{y}-$ y coordinate of the center of the circle segment,

$S_{z}-\mathrm{z}$ coordinate of the center of the circle segment,

$\alpha$ - crossing angle of profiles.

According to the respective analytical equations (1-5), the $\mathrm{z}$ coordinate components of the nodal point of both profiles are calculated. Based on the inputs entered, the program evaluates which of the nodal points is the desired result. This is followed by drawing of the result in form of an informative picture of the program dialog box. Coordinates are rounded to the required number of decimal places, converted to a text variable where the $y$ and $z$ coordinates are separated by a space. The decimal point is replaced with a dot. By doing so the nodal point coordinates are obtained and the process of generating them is repeated until the entire width of the composite profile is processed.

The generated coordinates can also be viewed directly in the program dialog box or saved to a text file. The last step before using it in a vehicle dynamics simulation program is to add a header and footer of the file that varies depending on the software used. Finally, it is necessary to overwrite the file extension, for example, for SIMPACK to * .prr for the rail profile or to * .prw for the profile describing the wheel geometry. 


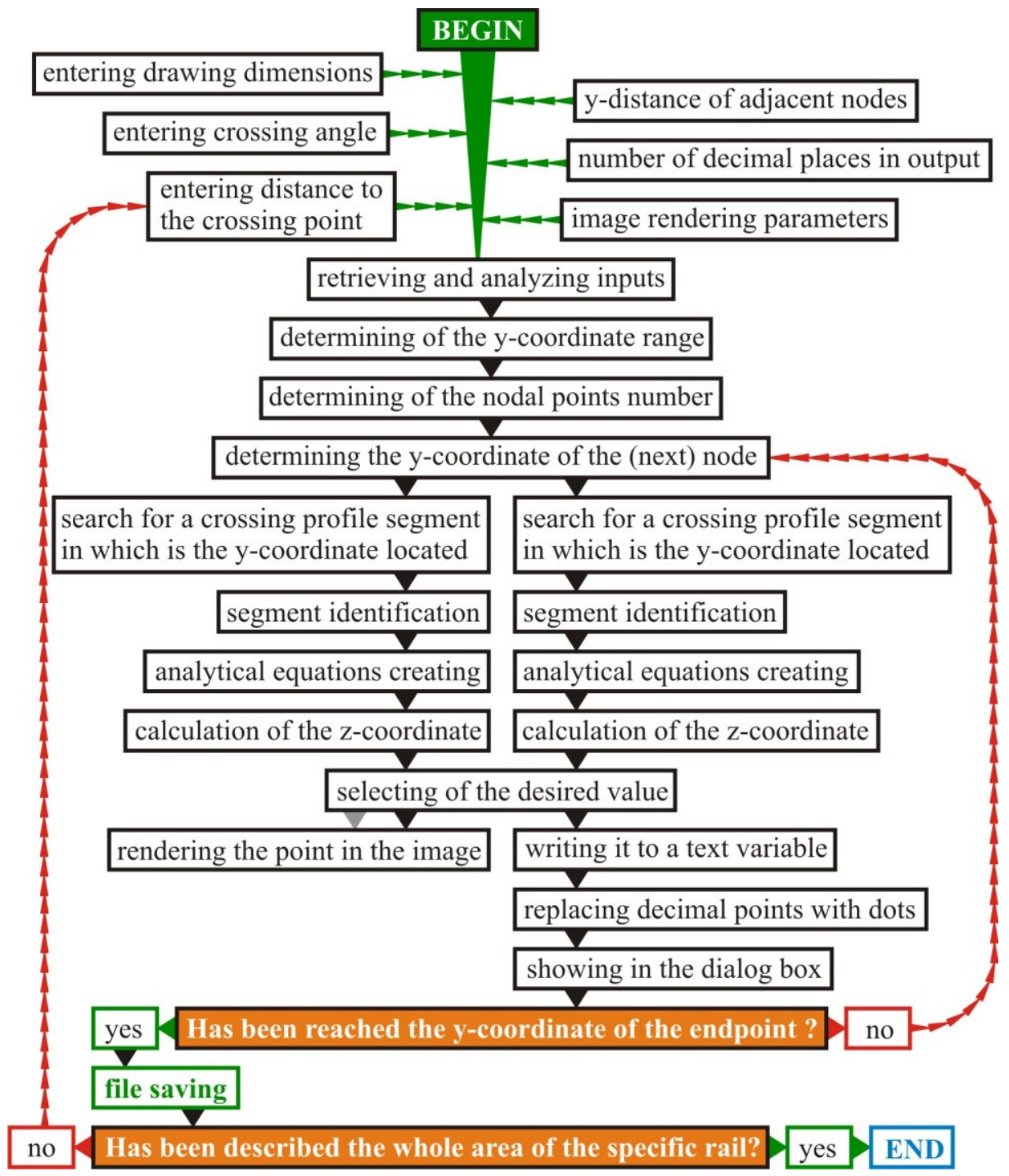

Fig. 5. Simplified schema describing the profile creation algorithm

\section{Example of using the program}

A program dialog box with the specified crossing profiles dimensions generating coordinates of the composite profile is shown in Fig. 6. Elements 1 and 7 as well as 8 and 9 serve to enter the specific dimensions of the direct or crossing profile from the drawing documentation. Elements 2 and 6 define the structure of output coordinates. Items 3 through 5 serve only for the graphical representation of the entered values by means of Figure 15. Elements 10 through 12 serve to define the position of the investigated point with respect to the point of crossing of the profiles. Element 13 determines the method of processing the overlapping parts of a straight and crossing profile. The coordinates generation process is triggered by the button 14 . The coordinates obtained can be viewed in window 16 and saved to the file using elements 17 and 18 . 


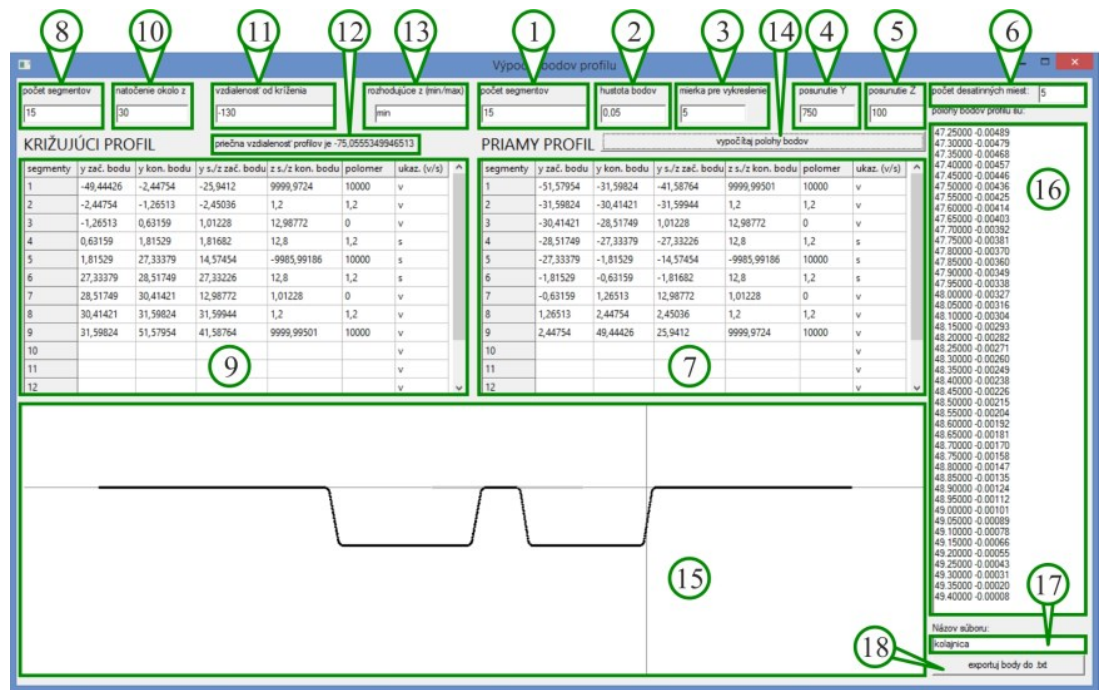

Fig. 6. Dialog box of the designed program

The files defining the crossing geometry obtained using the described application were entered into SIMPACK, where a simulation analysis of a ride of a tram through a point was carried out. Fig. 7 captures the right wheel of the first wheelset of the vehicle model at the point of the frog (Fig. 4).

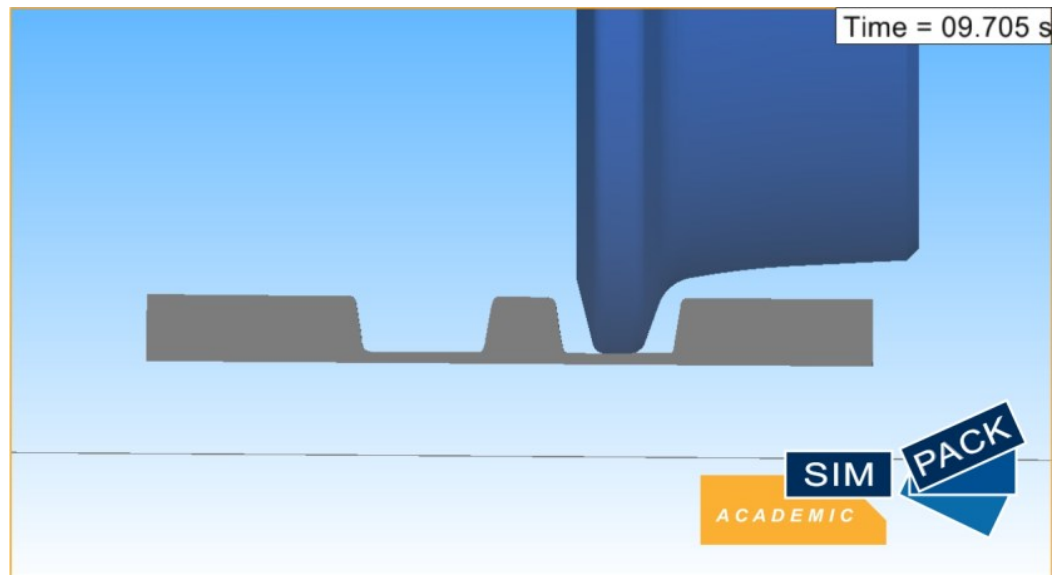

Fig. 7. Tram wheel when crossing the point frog in SIMPACK simulation program

\section{Conclusion}

In modelling the dynamics of a rail vehicle using modern multi-body software tools, the geometry of the profile of wheel and rail treads is one of the important input parameters. It is necessary to enter it in a form of files in the required form to ensure compatibility with the structure of inputs for the computational software, that is, in the form of coordinates of the points that can be used to translate the curve and thereby define the desired profile. In the article, the team of authors describes the methodology of the work of their own program enabling the efficient creation of files describing the geometry of the object based on its drawing documentation. It also provides an effective description of the variable geometry of the specific rails forming part of points, the shape of which is given by the composition 
of two profiles. This program was used, for example, to simulate the passage of a tramcar through a point frog and to design an atypical, small radius track arc with variable rail geometry. Solving this task required many times to repeat the process of creating profile files for the desired cross-sections of the rail, which would be rather lengthy without using the described program. The correctness of the program's work was validated by a problemfree cooperation of the obtained files with the SIMPACK computational program, in which a following simulation analysis of the dynamics of the vehicle's ride on a track with specific, in defined points variable profile of the rail belts was performed.

\section{References}

1. A. Chudzikiewicz, M. Opala, Application of computer simulation methods for running safety assessment of railway vehicles in example of freight cars. In Applied Mechanics and Materials, Vol. 9, pp 61-69. ISSN: 16609336 (2008)

2. J. Dižo, M. Blatnický, Use of multibody system dynamics as a tool for rail vehicle behaviour diagnostics. In Diagnostyka, Vol. 17, Iss. 2, pp. 9 - 16. ISSN:1641-6414 (2016)

3. J. R. Evans, T. K. Y. Lee, C. C. Hon, Optimising the wheel/rail interface on a modern urban rail system. In Vehicle System Dynamics. pp. 119-127. ISSN 1744-5159, (2009)

4. V. Tkachenko, S. Sapronova, I. Kulbovskyi, O. Fomin, Research into resistance to the motion of railroad undercarriages related to directing the wheelsets by a rail track. In Eastern European Journal of Enterprise Technologies. Vol. 5, Iss. 7-89, pp 65-72. ISSN: 17293774, (2017)

5. J. Gerlici, T. Lack, Wheelset/rail geometric characteristics assessment with regard to wheelset rolling. In Komunikacie, Vol. 7, Iss. 1, pp 5-10. ISSN: 13354205, (2006)

6. J. Gerlici, T. Lack, Iterative method for railway wheel profile design. In Komunikacie, Vol. 11, Iss. 2, pp 49-56. ISSN: 13354205 (2009)

7. A. Hába, J. Zelenka, M. Kohout, Determination of equivalent loading of turnout frog, Vibroengineering Procedia. Vol. 6, 10/2015, pp. 217-222 (2015)

8. T. Lack, J. Gerlici, Railway wheel and rail roughness analysis. In Komunikacie, Vol. 11, Iss. 2, pp 41-48. ISSN: 13354205 (2009)

9. V. Hauser, J. Gerlici, K. O. Kravchenko, T. Lack, M. Loulová, O. S. Nozhenko, O. P. Kravchenko, Determining coordinates of object's geometry profile points: Certificate of originality and copyright registration of the oeuvre. (UKRPATENT), 2017. 7 p. (2017)

10. V. Hauser, J. Gerlici, T. Lack, M. Loulová, O. S. Nozhenko, K. O. Kravchenko, O. V. Prosvirova, Railway bogie wheelset and curved track segment: Patent application no. a201701589 (In Ukrainian) Kyjiv : Ukrajinskyj instytut promyslovoji vlasnosti (UKRPATENT), 11 p. (2017)

11. SIMPACK 9.9.2 Documentation. 4556p. (2016)

12. L. Smetanka, P. Št'astniak, Analysis of contact stresses of theoretical and worn profile by using computer simulation. Manufacturing Technology, Vol. 17, Iss. 4, pp. 580585. ISSN: 12132489 (2017)

13. J A. Blaho, Informatics - Programming in Delphi (In Slovak). Bratislava: Slovenské pedagogické nakladatel'stvo. 144 p. ISBN 80-10-00421-9 (2006) 